\title{
Galactic dark matter halo made of spin-zero bosons
}

\author{
A. Bernal*, J. Barranco*, D. Alic ${ }^{\dagger, * *}$ and C. Palenzuela*,‡ \\ *Max-Planck-Institut für Gravitationsphysik, Albert Einstein Institut, 14476 Golm, Germany. \\ ${ }^{\dagger}$ Department of Physics, Universitat de les Illes Balears, Cra. Valldemossa, Spain. \\ ${ }^{* *}$ Currently at Max-Planck-Institut für Gravitationsphysik, Albert Einstein Institut, 14476 Golm, Germany \\ ${ }^{*}$ Currently at Canadian Institute of Theoretical Astrophysics, Toronto, Ontario, Canada
}

\begin{abstract}
Scalar Field Dark Matter models, in which the dark matter particle is a spin-0 boson, are becoming a serious alternative to the Cold Dark Matter paradigm. In the present work, we have constructed gravitational-bounded configurations made of massive spin zero bosons that can be used as models for galactic dark matter halos. These configurations are constructed in the context of Einstein's General Relativity and contrary to previous studies, where the bosons populate only the ground state, different excited states are coexisting simultaneously. We show that these 'mixed states' are stable under radial perturbations and that they provide more realistic rotation curves than single configurations.
\end{abstract}

Keywords: Dark matter, galactic halo, rotation curves

PACS: 04.40.-b,04.40.Dg,95.35.+d

\section{INTRODUCTION}

Despite the success of the $\Lambda$-cold dark matter scenario at cosmological scale, there is a strong controversy about its viability at galactic scale. Two predictions of this model that are still considered as unsolved problems are:

1. the cusp in the density profile of the dark matter (DM) for the galactic halos present at the center of the galaxy. This prediction is in contradiction with the flat profiles obtained by the analysis of the high resolution data of low surface brightness galaxies $[1,2]$ and,

2. the large number of satellite galaxies around each galactic halo, which exceeds far beyond what is observed around the Milky Way [3].

An alternative approach to $\Lambda-\mathrm{CDM}$ consists in describing the dark matter as a scalar field $[4,5,6,8]$. In this model, the dark matter particle is an ultra-light massive, spinless boson $\left(m \sim 10^{-23} \mathrm{eV}\right.$ [7]). A virtue of SFDM is that at cosmological scales it behaves as cold dark matter model [5, 4], but it doesn't suffer its defects at galactic scale: neither a cuspy profile [9], nor a over-density of satellite galaxies [10].

The formation of the galactic halo in the frame of the SFDM is as follows: as the universe expands, the scalar field cools together with the rest of the particles until it decouples from the rest of the matter. After that, only the expansion of the universe will keep cooling the scalar field. If a scalar field fluctuations are under the critical temperature, those will condensate leading to Boson-star (BS) like objects [11]. BSs are solutions of the Einstein-Klein-Gordon equations where the gravity attraction is balanced by the dispersive character of the scalar field. Considering the ultra-light mass of the dark matter particle, the boson's Compton wave length is of the order of kilo-parsecs, and the resulting BSs will look as structures with comparable length scales that could play the role of galactic halos.

Does this theoretical galactic halo reproduce the observed rotational curves? There have been several attempts in matching the theoretical predictions with the observational data using Newtonian BSs, where all the bosons are populating the same state, i.e. a ground or an excited state $[4,5,15]$. However, these models present problems, as the configurations in the ground state produce RCs which are not flat enough at large radii. On the other hand, RCs from excited BSs are in better agreement with the observed ones, but the excited BSs are known to be unstable [16, 17]. A promising alternative in modeling realistic dark matter halos consists in considering Newtonian configurations where bosons in the ground and excited states are coexisting [18]. Those configurations are stable and their RCs are flatter at large radii than those produced by BSs in the ground state [24].

The objective of this work is to study in more detail this alternative. First, we review the generalized relativistic BSs, the so called Multi-state Boson star (MSBS) [19], that are obtained when the scalar field is allowed to populate different energetic states. Special attention is pointed out in the stability of MSBS, as this is a necessary condition for 
MSBS to be considered viable astrophysical objects. Second, as we are interested in modeling galactic halos which can be considered Newtonian systems, we introduce the Newtonian limit of the relativistic MSBS and we show numerical evidence of their stability under radial perturbations. Finally, we construct RC for stable Newtonian MSBS that have a flat profile at large radii. In the present work, we are not considering the contribution of the baryonic matter to the RC. This is a reasonable assumption, as we compare these theoretical RC with the ones observed for low surface brightness galaxies which are mainly composed by dark matter.

\section{BOSON STARS AND MULTI STATE BOSON STARS}

BSs were initially introduced by Kaup [20] as solutions to the Einstein Klein-Gordon (EKG) equations, for a minimally coupled classical complex and massive scalar field. Ruffini and Bonazzola [21] constructed BSs as self-gravitating systems of spin-zero bosons. In this approach, the many particle system is described by a quantum real free field operator that satisfies the Klein-Gordon equation in a curved space-time. The metric coefficients of this space-time are solutions to the Einstein equations where the source term is given by the mean value of the energy momentum tensor operator constructed from the scalar field operator. This two approaches are equivalent as they yield to the same EKG equations and consequently the same macroscopic results [21]. Because BS are bounded solutions, regular everywhere and stable, they have been considered candidates of astrophysical objects in different contexts, depending mainly on the mass and the self-interaction potential of the scalar field. Two extensive reviews on BS are [22] and [23].

It was already pointed out in [21] that a possible generalization of BSs can be done by considering that the scalar field is not only populating a single state, but rather several states. This generalized relativistic MSBS were constructed and shown to be stable in [19]. The Newtonian limit of MSBS was considered in [18] and their stability under finite perturbations studied in [24]. Our current study, based on previous works, is focused on a comparison between the relativistic and Newtonian approaches in dealing with MSBS configurations. We present in the following subsections the equations that govern the behavior of MSBS and the procedures used for obtaining solutions to these equations. This study allows us to draw conclusions about the regime in which MSBS are well described using the Newtonian limit.

\section{General relativistic MSBS}

We start by considering the semiclassical limit of the Einstein equations

$$
G_{\alpha \beta}=8 \pi G\left\langle Q\left|\hat{T}_{\alpha \beta}\right| Q\right\rangle,
$$

where $\left\langle Q\left|\hat{T}_{\alpha \beta}\right| Q\right\rangle$ denotes the mean value of the stress energy tensor $\hat{T}_{\alpha \beta}$ over a system of bosons which are in the state $|Q\rangle$. The many-particle system is described by the real, second quantized scalar field

$$
\hat{\Phi}=\sum_{n l m}\left[\hat{b}_{n l m} \Phi_{n l m}(t, \mathbf{x})+\hat{b}_{n l m}^{\dagger} \Phi_{n l m}^{*}(t, \mathbf{x})\right]
$$

The operators $\hat{b}_{n l m}$ and $\hat{b}_{n l m}^{\dagger}$ are the usual creation and respectively annihilation operators which satisfy the commutation relations $\left[\hat{b}_{n l m}^{\dagger}, \hat{b}_{n^{\prime} l^{\prime} m^{\prime}}^{\dagger}\right]=\left[\hat{b}_{n l m}^{-}, \hat{b}_{n^{\prime} l^{\prime} m^{\prime}}^{-}\right]=0$ and $\left[\hat{b}_{n l m}^{\dagger}, \hat{b}_{n^{\prime} l^{\prime} m^{\prime}}^{-}\right]=\delta_{n n^{\prime}} \delta_{l l^{\prime}} \delta_{m m^{\prime}}$.

From operator $\hat{\Phi}$ it is possible to construct the energy-momentum tensor operator $\hat{T}_{\mu v}$ just by inserting eq. (2) into the classical expression for the energy-momentum tensor. The state $|Q\rangle=\left|N_{100}, N_{200} \ldots N_{n l m} \ldots\right\rangle$ is composed of many scalar particles distributed in sets of $N_{n l m}$ particles of mass $\mu$, angular momentum $l$ and azimuthal momentum $m$.

Due to orthogonality of the quantum states, it is found that

$$
\left\langle Q\left|\hat{T}_{\alpha \beta}\right| Q\right\rangle=\sum_{n=1}^{\infty} \sum_{l=1}^{n-1} \sum_{m=-l}^{l}\left\langle N_{n l m}\left|\hat{T}_{\alpha \beta}\right| N_{n l m}\right\rangle,
$$

where $\left|N_{n l m}\right\rangle=\left|0,0, \ldots N_{n l m} . .0,0\right\rangle$, i.e. the stress energy tensors of the system is the linear superposition of the expectation values of the energy-momentum tensor for each individual state. Finally, the Einstein equations (1) with the stress energy tensor (3) can be written as

$$
G_{\alpha \beta}=16 \pi G \sum_{n, l, m} N_{n l m} T_{\alpha \beta(n l m)} .
$$



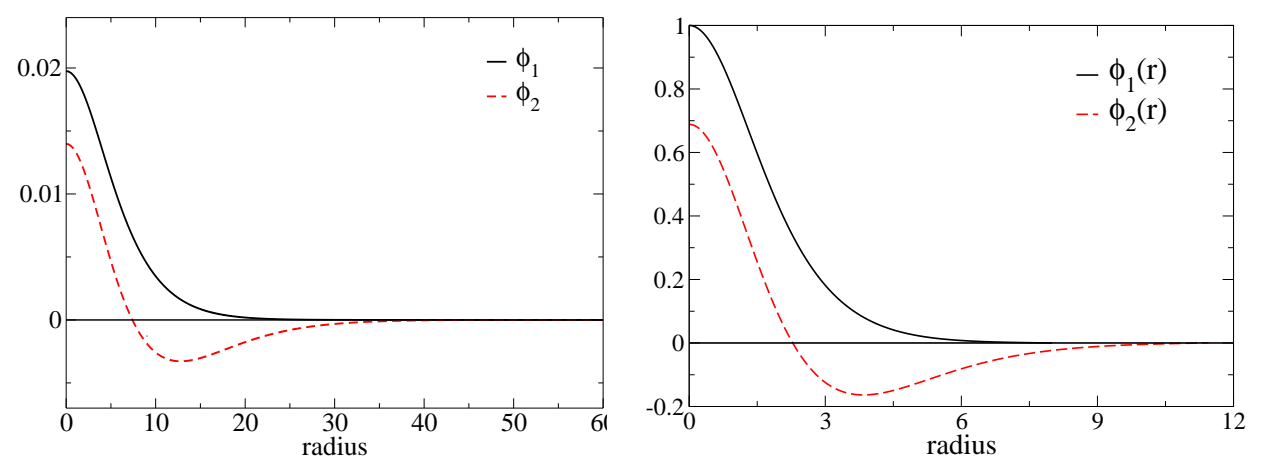

FIGURE 1. Typical solutions to the relativistic MSBS (eqs. 8a) and Newtonian MSBS (eqs. 9) configurations. In both cases the number of excited particles $N_{2}$ is equal to the number of ground state particles $N_{1}$.

On the other hand, we are dealing with a system of bosons $\hat{\Phi}$ which satisfy the Klein-Gordon equation, and due to eq. (2) each field coefficient satisfies its own Klein-Gordon equation:

$$
\left(\square-\mu^{2}\right) \Phi_{n l m}(t, \mathbf{x})=0 .
$$

where $\square=(1 / \sqrt{-g}) \partial_{\mu}\left[\sqrt{-g} g^{\mu v} \partial_{v}\right]$ and $\mu$ is the mass of the scalar field.

Summarizing, we have shown that in the case where particles populate various excited levels, the source of the Einstein equations (4) is equivalent to the energy momentum tensor of many (independent) classical complex scalar fields $\Phi_{n l m}(t, \mathbf{x})$ minimally coupled to gravity. Each one of these scalar fields accounts for only one of the excited single states $\left|N_{n l m}\right\rangle$, and its dynamics is given by its own KG equation (5).

In the spherically symmetric case, $l=m=0$, when the fields have an harmonic dependence

$$
\sqrt{8 \pi G} \Phi_{n l m}(t, \mathbf{x}) \rightarrow e^{-i \omega_{n} t} \phi_{n}(r)
$$

the line element can be written as

$$
d s^{2}=-\alpha^{2}(r) d t^{2}+a^{2}(r) d r^{2}+r^{2} d \Omega .
$$

Then the Einstein eqs. (4) and the KG eqs. (5) take the form

$$
\begin{aligned}
\partial_{r} a & =\frac{a}{2}\left\{-\frac{a^{2}-1}{r}+4 \pi r \sum_{n=1}^{\mathscr{I}}\left[\left(\frac{\omega_{n}^{2}}{\alpha^{2}}+m^{2}\right) a^{2} \phi_{n}^{2}+\Phi_{n}^{2}\right]\right\}, \\
\partial_{r} \alpha & =\frac{\alpha}{2}\left\{\frac{a^{2}-1}{r}+4 \pi r \sum_{n=1}^{\mathscr{I}}\left[\left(\frac{\omega_{n}^{2}}{\alpha^{2}}-m^{2}\right) a^{2} \phi_{n}^{2}+\Phi_{n}^{2}\right]\right\}, \\
\partial_{r} \phi_{n} & =\Phi_{n}, \\
\partial_{r} \Phi_{n} & =-\left\{1+a^{2}-4 \pi r^{2} a^{2} m^{2}\left(\sum_{s=1}^{\mathscr{g}} \phi_{s}^{2}\right)\right\} \frac{\Phi_{n}}{r}-\left(\frac{\omega_{n}^{2}}{\alpha^{2}}-m^{2}\right) \phi_{n} a^{2} .
\end{aligned}
$$

where the indexes $n$ and $s$ run from 1 to $\mathscr{I}, \mathscr{I}$ the number of states populated in the MSBS.

In order to obtain a solution of this system, we impose as boundary conditions regularity at origin and asymptotic flatness for the metric components.

\section{Newtonian MSBS}

The Newtonian limit of the coupled Einstein-Klein-Gordon (EKG) equations (4-5) is the so-called SchrödingerPoisson (SP) system [27]

$$
\begin{aligned}
\nabla^{2} U & =\sum_{n l m}\left|\Psi_{n l m}\right|^{2}, \\
i \partial_{t} \Psi_{n l m} & =-\frac{1}{2} \nabla^{2} \Psi_{n l m}+U \Psi_{n l m},
\end{aligned}
$$


where $\Psi_{n l m}$ is related to $\Phi_{n l m}$ by

$$
\sqrt{8 \pi G} \Phi_{n l m}(t, \mathbf{x})=e^{-i \mu t} \Psi_{n l m}(t, \mathbf{x}) .
$$

Then, the Newtonian version of the EKG equations describes the dynamics of the non-relativistic parts of the scalar fields, which are coupled among themselves through the Newtonian gravitational potential $U$ [25]. We constructed solutions to the SP system (9) when $\mathscr{N}$ bosons are allowed to occupy $\mathscr{I}$ different levels, which for simplicity of the discussion will have zero angular momentum $(l=0, m=0)$. Hence, the states are of the form $|Q\rangle=\left|N_{1}, N_{2}, N_{3}, \ldots, N_{\mathscr{I}}\right\rangle$. We are assuming spherical symmetry and an harmonic time dependence for the scalar field $\Psi_{n}=e^{-i \omega_{n} t} \phi_{n}(r)$. Then the system (9) reads

$$
\begin{aligned}
\frac{1}{r^{2}} \frac{d^{2}\left(r^{2} \phi_{n}\right)}{d r^{2}} & =\left(U+\omega_{n}\right) \phi_{n}, \\
\frac{1}{r^{2}} \frac{d^{2}\left(r^{2} U\right)}{d r^{2}} & =\sum_{n=1}^{\mathscr{I}}\left|\phi_{n}\right|^{2} .
\end{aligned}
$$

Given appropriate boundary conditions in order to obtain regular and bounded solutions, the system (11) becomes an eigenvalue problem for the temporal frequencies $\omega_{n}$.

\section{Solving MSBS}

Starting from the semiclassical limit of the Einstein equations, we have derived the static, spherically symmetric equations for the case when a massive spinless boson with different and coexisting energy states is considered. We have arrived to the general relativistic Einstein-Klein-Gordon equations 8a and from that system, we deduced the Newtonian limit of those equations arriving to the Schrödinger-Poisson system eqs. 9. There is no analytical solution except to the case where the scalar field is massless. However we are interested in the self-gravitating system and the only way of obtaining solutions is through numerical solutions. The solutions were calculated numerically using finite differencing and a shooting routine that searched $\omega_{n}$. Typical solutions are shown in Fig. 1. Details of the equilibrium configurations for the particular case when only two states are considered, the ground and the first excited state, can be found in [19] and in [24] for the relativistic MSBS and the Newtonian MSBS respectively.

\section{Equivalence of MSBS $v s$. Newtonian MSBS}

The complete description of MSBS is given by the Einstein equations while the Newtonian equations provide a reasonable approximation in some cases. Nevertheless, it is a good cross-check exercise to verify that under the specific conditions, relativistic MSBS and Newtonian MSBS coincide.

A quantity that measures the importance of relativistic corrections is the compactness of the object. In Fig. 2 the compactness of relativistic equilibrium BS in the ground state is shown. This compactness is defined as $2 M / R_{99}$ where $M$ is the BS's ADM mass and $R_{99}$ is the radius containing $99 \%$ of the total particle number. In the definition above, we consider $R_{99}$ instead of the physical radius of the star which extends to infinity, as we consider that $R_{99}$ is a reasonable distance where for measuring the gravitational field of the star.

It is clear from Fig. 2 that Newtonian configurations which are expected to have a very small compactness (as a reminder, the compactness of the Sun is $\sim 10^{-5}$ ) corresponds to the those solutions for which the central value of the scalar field satisfies the condition $\phi(0) \ll 1$.

We have computed configurations of two-state boson stars, solving the full relativistic EKG equations (8a), for which the number of particles in the ground state is equal to the number of particles in the first excited state and with the central value of the ground state $\phi_{1}(0) \ll 1$. In Fig. 3 we show the ADM mass of these configurations and compare it with the mass of the corresponding configurations obtained by solving the Schrödinger-Poisson system (11). We found no difference between those masses for small values of $\phi_{1}(0)$. Fig. 3 shows the radial functions of the scalar fields for the populated states $\phi_{1}(r)$ and $\phi_{2}(r)$ obtained from the EKG system and from the SP systems. In the same figure, the Newtonian potential $U(r)$ is compared against $a(r)-1$ which is the "equivalent" potential for the general relativistic case. From this set of plots shown in Fig. 3 we can conclude that Newtonian solutions describe with a good approximation MSBS with small values of the scalar field in the center. 


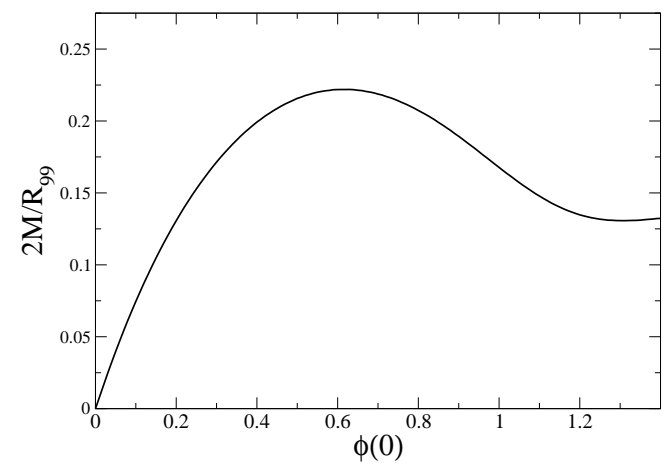

FIGURE 2. Compactness $\left(M / R_{99}\right)$ of $\mathrm{BS}$ in the ground state.
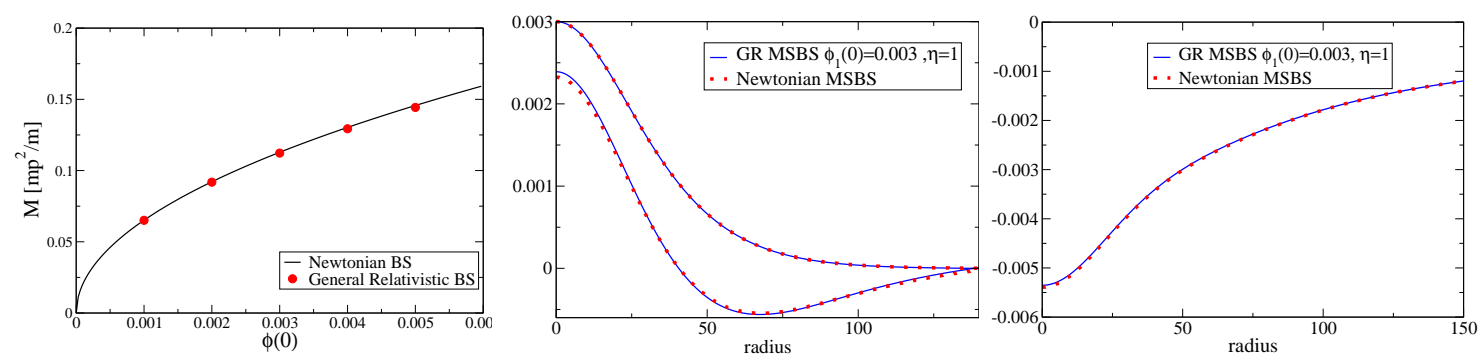

FIGURE 3. Left: Total mass for relativistic ground state BS and Newtonian ground state BS. Center: $\phi_{1}(r)$ and $\phi_{2}(r)$ for relativistic MSBS (solid line) and Newtonian MSBS (dotted line) for $\phi_{1}(0)<<1$ and $\eta=1$. Right: $a(r)-1$ of a relativistic MSBS (solid line) and Newtonian potential $U(r)$ (dotted line) for $\phi_{1}(0)<<1$ and $\eta=1$.

\section{STABILITY OF MSBS}

In order to extend the importance of the MSBS beyond the mathematical context, it is first necessary to prove their stability. The previous stability studies of BS can be classified in two types:

1. studies that consider infinitesimal perturbations for which the number of particles is conserved and,

2. studies that consider finite perturbations and there is no conservation in the number of particles.

In the first type of studies the methods employed have been linear perturbation theory $[29,30,16]$ and catastrophe theory [31]. On the other hand, when finite perturbations are considered, the final state of the perturbed BS can be known only through its numerical evolution. Furthermore, the results obtained for infinitesimal perturbations have been cross-checked using numerical techniques [32, 17, 33].

From those studies, it is known that BSs in the ground state are stable against radial perturbations if the amplitude of the scalar field at the origin $\phi(0)$ is smaller than a critical value $\phi_{c}(0)$, for which the maximum mass $M_{\max }$ is reached. However in the case of excited BSs, namely those in which the scalar field is in an excited state, there are some differences: if infinitesimal perturbations are considered, excited BSs with $\phi(0)$ smaller than the central value of the field for which the maximum mass is reached, are stable [16, 22]. On the other hand, under finite perturbations, excited BSs are intrinsically unstable even for $\phi(0) \leq \phi_{c}(0)$, since these perturbations drive the star either into a ground state $\mathrm{BS}$, or into a black hole [16, 17].

Since MSBS contains at least one excited state, one could infer that MSBS would be unstable under finite perturbations. The stability of relativistic MSBS composed of two states, the ground and the first excited state, was studied in [19] using numerical techniques. These two-state boson stars are characterized by: $i$ ) the fraction $\eta=N_{2} / N_{1}$, where $N_{2}$ is the number of particles in the excited state and $N_{1}$ is the number of particles in the ground state, and ii) the central amplitude of one of the scalar fields (either $\phi_{1}(0)$ or $\phi_{2}(0)$ ). A set of MSBS with different values of $\eta$ was selected for 

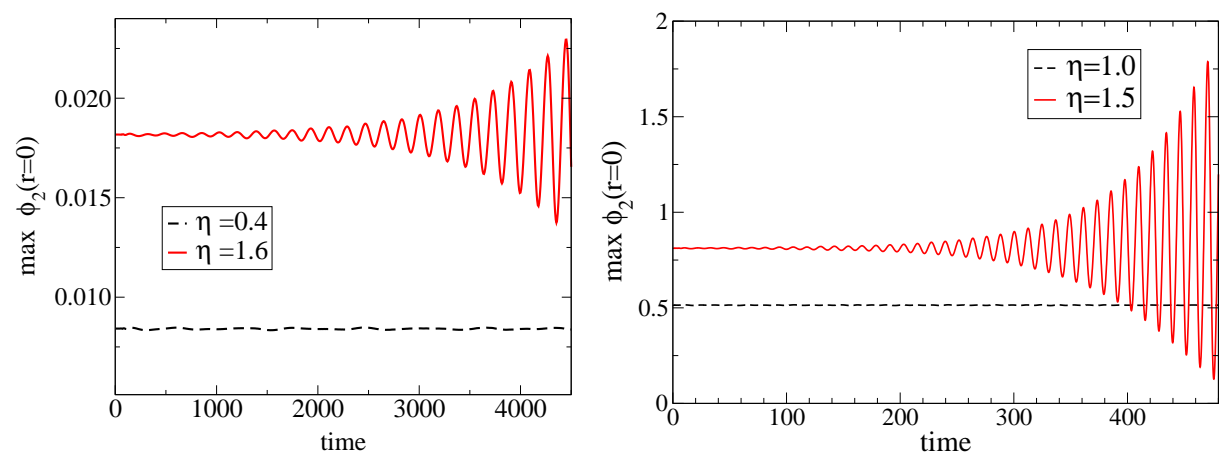

FIGURE 4. Left: Time evolution of $\phi_{2}(r=0)$ for two perturbed relativistic MSBS (solid line $\eta=1.6$, dashed line $\eta=0.4$ ). Right: Time evolution of $\phi_{2}(r=0)$ for two perturbed Newtonian MSBS (solid line $\eta=1.5$, dashed line $\eta=1.0$ ).

this numerical stability study.

The perturbation consisted in an additional massless scalar field, which contains $0.01 \%$ of the total energy density of the MSBS. The numerical evolution of the perturbed configurations shown that for MSBS with $\eta>1$ the perturbations grow exponentially. However, for configurations with $\eta<1$, the perturbations only oscillate without growing. Comparing these two behaviors it is possible to infer that two-state BSs with $\eta<1$ are stable, while those with $\eta>1$ are not. A systematic study was done perturbing configurations with different values of $\eta$ in order to find the maximum fraction for which the configurations can be stable and it was found that $\eta_{\max } \approx 1$.

The first plot in Fig. 4 shows the central value of the excited scalar field $\phi_{2}(r=0, t)$, for the cases $\eta=0.4$ and $\eta=1.6$. These models exhibit different It is clear that there are two different behaviors: for $N_{2}>N_{1}$ there is an exponential growth of the amplitude, while for $N_{2}<N_{1}$ the amplitude of $\phi_{2}(r=0, t)$ remains constant, despite the fact that both have been affected by the same type of finite perturbation. More details about those results and about the fate of the unstable configurations can be found in [19].

In [24], a similar study regarding the stability of two-state BS in the Newtonian limit was performed and the results of the stability conditions for those configurations are consistent with the obtained for their relativistic counterparts. This comparison can be seen in the right plot of Fig. 4 which shows the temporal behavior of $\phi_{2}(r=0, t)$ for configurations with $\eta=1.0$ and $\eta=1.5$, namely stable and respectively unstable Newtonian MSBS.

\section{ROTATION CURVES FROM MSBS}

In this section, we show that mixed ground-excited states produce flat rotation curves at large radii, so they can be considered realistic models for dark matter halos $[18,24]$

A typical DM halo mass is $\sim 10^{12} \mathrm{M} \odot$ and its radius is $R \sim 100 \mathrm{Kpc}$ therefore its compactness is

$$
\frac{2 M}{R_{99}} \sim 10^{-7}
$$

then it is reasonable to consider the halo as a Newtonian system and therefore, in accordance with our previous discussion, Newtonian MSBS should be appropriate in modeling it.

We calculate the velocity of test particles moving along circular orbits in the gravitational potential sourced by the MSBS configurations via the Newtonian formula

$$
v(r)=\sqrt{\mathscr{N}(t, r) / r},
$$

where $\mathscr{N}(t, r)$ is the total number of particles inside the radius $r$ obtained from the numerical equilibrium configurations of the SP system (11).

The results for a MSBS with $\eta=1$ are shown in Fig. 5. We can see a significant improvement in the flatness of the rotation curve at large radii compared when compared with the RC for a BS in the ground state.

Nevertheless, there is room for further improvements. For example, we can consider a MSBS with the ground, first and second states populated, and compute its associated rotation curve, as presented in Fig. 5. One can notice that the configurations with more excited states lead to flatter RC. 


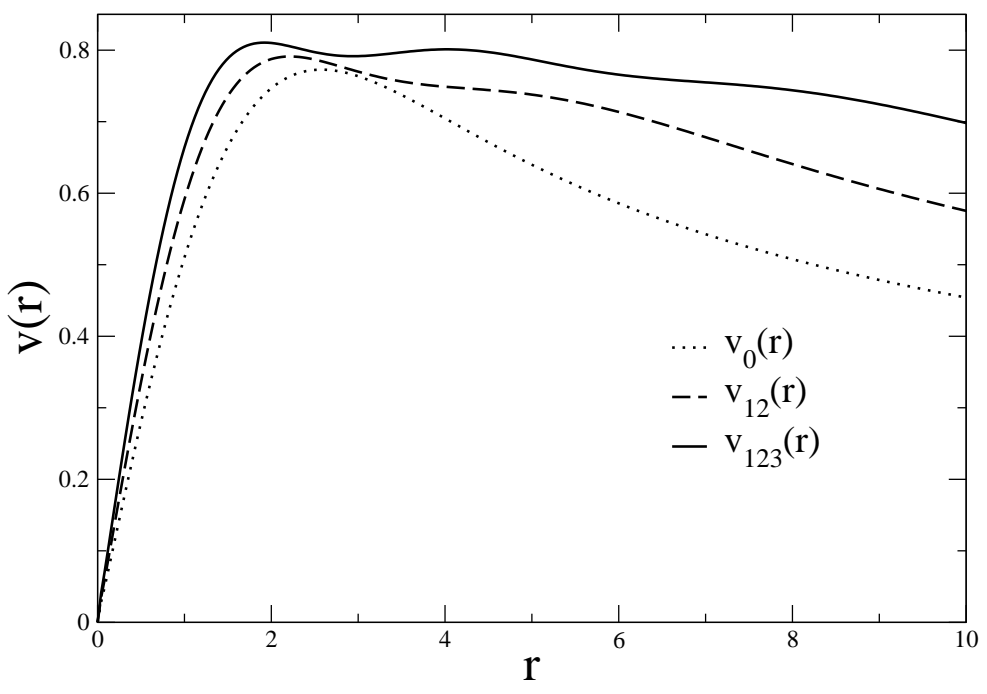

FIGURE 5. Rotation curve for: ground state BS (dotted line), Newtonian MSBS with two states and $\eta=1$ (dashed line) and Newtonian MSBS with three states and $N_{1}>N_{2}>N_{3}$ (solid line).

There is another particular feature we would like to mention. The introduction of multi states provides additional free parameters which allow a better fit to the observed rotation curves. The extra parameters are the occupation numbers of the mixed state, namely $N_{1}, N_{2}, N_{3}$, etc., which are related to the $\Psi_{n}(0,0)$ values. These values could be determined by the local environment to which the scalar halo was subjected during its formation.

\section{ACKNOWLEDGMENTS}

$\mathrm{AB}$ and JB thank L. Rezzolla for his support and hospitality at the AEI. AB and JB are partially supported by CONACyT Mexico.

\section{REFERENCES}

1. S. S. McGaugh, V. C. Rubin and W. J. G. de Blok, Astron. J. 122, 2381 (2001) [arXiv:astro-ph/0107326].

2. B. Moore, T. R. Quinn, F. Governato, J. Stadel and G. Lake, Mon. Not. Roy. Astron. Soc. 310, 1147 (1999) [arXiv:astro$\mathrm{ph} / 9903164]$.

3. A. A. Klypin, A. V. Kravtsov, O. Valenzuela and F. Prada, Astrophys. J. 522, 82 (1999) [arXiv:astro-ph/9901240].

4. V. Sahni and L. M. Wang, Phys. Rev. D 62, 103517 (2000) [arXiv:astro-ph/9910097].

5. T. Matos and L. A. Urena-Lopez, Class. Quant. Grav. 17, L75 (2000) [arXiv:astro-ph/0004332].

6. W. Hu, R. Barkana and A. Gruzinov, Phys. Rev. Lett. 85, 1158 (2000) [arXiv:astro-ph/0003365].

7. L. A. Urena-Lopez and T. Matos, Phys. Rev. D 62, 081302 (2000) [arXiv:astro-ph/0003364].

8. P. J. E. Peebles, arXiv:astro-ph/0002495.

9. T. Matos and D. Nunez, arXiv:astro-ph/0303455.

10. T. Matos and L. A. Urena-Lopez, Phys. Rev. D 63, 063506 (2001) [arXiv:astro-ph/0006024].

11. T. Matos, J. A. Vazquez and J. Magana, arXiv:0806.0683 [astro-ph].

12. S. J. Sin, Phys. Rev. D 50, 3650 (1994) [arXiv:hep-ph/9205208].

13. S. U. Ji and S. J. Sin, Phys. Rev. D 50, 3655 (1994) [arXiv:hep-ph/9409267].

14. F. S. Guzman and L. A. Urena-Lopez, Astrophys. J. 645, 814 (2006) [arXiv:astro-ph/0603613].

15. A. Arbey, J. Lesgourgues and P. Salati, Phys. Rev. D 68, 023511 (2003) [arXiv:astro-ph/0301533].

16. T. D. Lee and Y. Pang, Nucl. Phys. B 315, 477 (1989).

17. J. Balakrishna, E. Seidel and W. M. Suen, Phys. Rev. D 58, 104004 (1998) [arXiv:gr-qc/9712064].

18. T. Matos and L. A. Urena-Lopez, Gen. Rel. Grav. 39, 1279 (2007).

19. A. Bernal, J. Barranco, D. Alic and C. Palenzuela, arXiv:0908.2435 [gr-qc].

20. D. J. Kaup, Phys. Rev. 172, 1331 (1968).

21. R. Ruffini and S. Bonazzola, Phys. Rev. 187, 1767 (1969). 
22. P. Jetzer, Phys. Rept. 220, 163 (1992).

23. F. E. Schunck and E. W. Mielke, Class. Quant. Grav. 20, R301 (2003) [arXiv:0801.0307 [astro-ph]].

24. A. Bernal, L. Urena-Lopez In preparation.

25. F. S. Guzman and L. A. Urena-Lopez, Phys. Rev. D 69, 124033 (2004) [arXiv:gr-qc/0404014].

26. R. Friedberg, T. D. Lee and Y. Pang, Phys. Rev. D 35, 3658 (1987).

27. R. Friedberg, T. D. Lee and Y. Pang, Phys. Rev. D 35, 3640 (1987).

28. R. Friedberg, T. D. Lee and Y. Pang, Nucl. Phys. B 276, 549 (1986).

29. M. Gleiser, Phys. Rev. D 38, 2376 (1988) [Erratum-ibid. D 39, 1258 (1989)].

30. M. Gleiser and R. Watkins, Nucl. Phys. B 319, 733 (1989).

31. F. V. Kusmartsev, E. W. Mielke and F. E. Schunck, Phys. Rev. D 43, 3895 (1991) [arXiv:0810.0696 [astro-ph]].

32. E. Seidel and W. M. Suen, Phys. Rev. D 42, 384 (1990).

33. S. H. Hawley and M. W. Choptuik, Phys. Rev. D 62, 104024 (2000) [arXiv:gr-qc/0007039]. 